\title{
Fabrication and Mechanical Characterization of Water-Soluble Resin-Coated Natural Fiber Green Composites*
}

\author{
Ken-ichi MANABE** and Tomoyuki HAYAKAWA*** \\ **Department of Mechanical Engineering, Tokyo Metropolitan University \\ 1-1 Minamiosawa, Hachioji-shi, Tokyo, Japan \\ E-mail:manabe@tmu.ac.jp \\ ***Department of Mechanical Engineering, Tokyo Metropolitan University \\ 1-1 Minamiosawa, Hachioji-shi, Tokyo, Japan \\ (Presently, Mitsubishi Heavy Industries, Ltd)
}

\begin{abstract}
In this study, water-soluble biodegradable resin was introduced as a coating agent to improve the interfacial strength and then to fabricate a high-performance green composite with polylactic acid (PLA) and hemp yarn. Dip coating was carried out for hemp yarn and the green composites were fabricated by hot processing. The coated green composite achieves a high tensile strength of $117 \mathrm{MPa}$ even though the fiber volume fraction is less than $30 \%$. Interfacial shear strength (IFSS) was measured by a single fiber pull-out test, and the effect of water-soluble resin on the tensile properties of the composites was evaluated. As a result, when using coated natural bundles, the IFSS value is smaller than when using noncoated natural bundles. On the basis of observations of the fractured surface of composites and initial yarns using a scanning electron microscope (SEM), the effect of the impregnation of water-soluble resin into the natural bundles on the tensile strength is discussed in detail. It is found that water-soluble resin is effective in improving the mechanical properties of the composite, although the interfacial strength between PLA and water-soluble resin was decreased, and as a result, the tensile strength of green composites increases by almost $20 \%$.
\end{abstract}

Key words: Green Composites, Natural Fiber, Water-Soluble Resin, Interfacial Shear Strength, Tensile Property, Coating Agent

\section{Introduction}

In the last decade, ecofriendly fiber reinforcement composite materials, green composites, which consist of biodegradable resin such as polylactic acid (PLA) as a matrix and natural fiber such as hemp or bamboo as reinforcements, have attracted attention in efforts to reduce the environmental load in the world. Conventional composites such as glass-fiber-reinforced plastic (GFRP) and carbon-fiber-reinforced plastic (CFRP) possess high environmental burden so that green composites have been expected as substitute materials of high-environmental-burden fiber-reinforced plastics.

In many previous studies on green composites, natural fibers such as flax ${ }^{(1)-(3)}$, ramie $^{(1)}$, jute $^{(1)(4)}$, bamboo ${ }^{(5)}$, pineapple ${ }^{(6)}$, kenaf $^{(7)}$, henequen ${ }^{(8)}$, hemp ${ }^{(9)(10)}$, and maize ${ }^{(11)}$ were used as reinforcements in green composites containing biodegradable polymers. All such 
composites had a fiber volume fraction $V_{\mathrm{f}}$ of less than $55 \%$ and were molded at high temperatures of $180{ }^{\circ} \mathrm{C}$ and $210^{\circ} \mathrm{C}$, although for polycaprolactone (PCL) matrix, they were done at $130^{\circ} \mathrm{C}$ in the case of maize ${ }^{(11)}$. Because of the above reasons, these composites had tensile and flexural strengths lower than $120 \mathrm{MPa}$. As a result, presently, the application of green composites has been limited to interior materials and various functional materials, owing to their lower mechanical strength. In general, the fiber/matrix interface characteristic related to the transmission of applied stresses to the reinforcement fiber has a particularly strong effect on the strength characteristics.

To further enhance the strength characteristics, we should focus on the characteristics of the interface between the fiber/yarn and the matrix of green composites. However, because the present biodegradable resins generally have high molten viscosity and low wettability, it is difficult to impregnate resin among the reinforcement fibers; such inferior interface conditions result in pullout and detachment of the fibers from the matrix, disturbing the effective improvement in the strength and volume fraction $V_{\mathrm{f}}$ of the composite.

To solve these problems of low $V$ and low strength, Ochi ${ }^{(10)}$ proposed the use of water-soluble biodegradable resin as the matrix and confirmed its validity. The developed green composites, which contain the water-soluble biodegradable resin and manila hemp fiber bundles $V_{\mathrm{f}}$ of up to $70 \%$, have a high strength of $365 \mathrm{MPa}$. This is a special case and the application is limited to green composites with only the water-soluble resin matrix. However, Ochi's result implies that the water-soluble resin would be effective as an interfacial-conditioning agent. Therefore, water-soluble resins would be better used as coating agents in order to expand the range of application of such composites.

In this study, water-soluble biodegradable resin is focused on to enhance the surface characteristic of the fiber/matrix interface for green composites. A water-soluble biodegradable polyvinyl alcohol (PVA) is used as a coating agent for natural fiber bundles, a tensile specimen of the composite is made by hot pressing, and the tensile test is carried out to evaluate the tensile characteristics. Through the test, the improvement of the interfacial characteristics and tensile property of the composite with PLA and hemp yarn is confirmed for the fabrication of high-strength green composite. The interfacial shear strength (IFSS) between the constituents is measured by a single fiber pull-out test. In addition, observation by scanning electron microscope (SEM) is conducted to clarify the effect of the impregnation of water-soluble resin into yarns/fibers on the tensile strength.

\section{Experiment}

\subsection{Materials used}

PLA resin (Shimadzu Corporation, LACTY \#9010) is adopted as the biodegradable matrix. This resin is prepared in pellet form. As the natural fiber bundles, hemp fiber bundles made through spinning and weaving processes are used. A bundle strand used in this study contains twenty fibers. As a coating agent of the natural fiber bundles, water-soluble resin PVA (Kuraray, PVA-505) is used in aqueous solution with a mass content of $20 \%$.

\subsection{Tensile test of hemp bundles and resin matrix}

Figure 1 shows the schematic illustration of a tensile specimen with a paperboard support prepared to prevent damage to the hemp bundles during its handling. The cross-sectional area of a bundle strand was calculated from their circular-assumed diameters simply measured using a micrometer. Because of the measuring pressure of the micrometer, the bundle crosssection is slightly deformed elliptically, whereby a more valid cross-sectional area of a bundle strand without any gap space could be calculated; this value 
was not statistically different from the value obtained by optical cross-sectional measurement. A hemp bundle was glued to the paperboard support using epoxy resin, carefully gripped by the testing machine, and then the center of the paperboard support was cut. The tensile test was performed at a crosshead speed of $1.0 \mathrm{~mm} / \mathrm{min}$.

For the resin matrix, a tensile specimen was prepared in the same way as in the case of the green composite described in the next section.

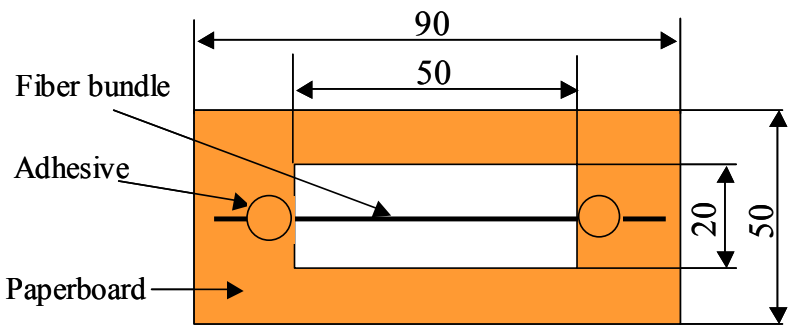

[Unit:mm]

Fig. 1 Schematic of tensile specimen for a fiber bundle.

\subsection{Preparation of green composite tensile specimen and tensile test conditions}

First, hemp fiber bundles are dipped in an aqueous solution of water-soluble PVA for three hours. After they are removed from the solution, they are dried in atmosphere for 24 hours, at which time the impregnation treatment and coating of natural fiber bundles are complete. Next, to fabricate the composite tensile specimen, a hot pressing method ${ }^{(1)}$ is adopted in this experiment. Firstly, LACTY pellets are put into a mold for the tensile specimen and heated and pressed at $180{ }^{\circ} \mathrm{C}$ for about 20 minutes. After that, the natural fiber bundles coated with water-soluble resin and pellet-form LACTY are placed into the mold and reheated for around 30 minutes until completely melted. The molding pressure of 2 $\mathrm{MPa}$ is applied in the molding stage using a universal hydraulic testing machine. The geometry and dimensions of the tensile specimen are in accordance with ASTM Code D 638 (Type V) as shown in Fig.2. Two kinds of tensile specimens, a composite tensile specimen with coated fiber bundles (treated hemp composite, THC) and one with non-coated fiber bindles (untreated hemp composite, UTHC), are prepared to confirm the effect of interfacial strength improvement. The volume fraction of fiber in the case of THC is $24 \%$ and $27 \%$ for UTHC.

To evaluate the fundamental mechanical properties of the green composites, tensile testing was performed at a crosshead speed of $1.0 \mathrm{~mm} / \mathrm{min}$ using a universal testing machine at room temperature.

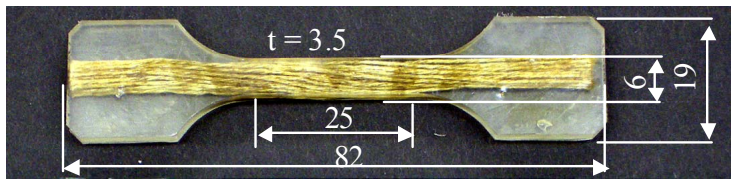

[Unit:mm]

Fig. 2 Geometry and dimensions of tensile test specimen of matrix and fiber-reinforced composite. 


\subsection{Preparation of pull-out test specimen of yarn and its test conditions}

The test specimen for the pull-out test of yarn is fabricated by hot pressing using a mold with a specified primary shape. Firstly, both ends of a yarn for the primary test specimen are free and outside the molded matrix. Using this primary molded specimen, both ends are cut off and finished to specified dimensions using a high-speed precision fine cutting machine, as shown in Fig. 3(a). After that, the end face is polished carefully so that the length of yarn embedded in the matrix $L$ is about $850 \mu \mathrm{m}$.

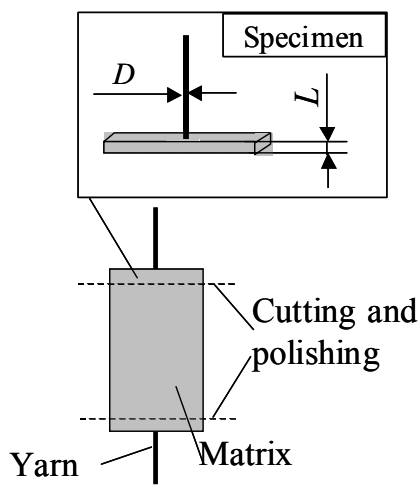

(a) Pull-out test specimen

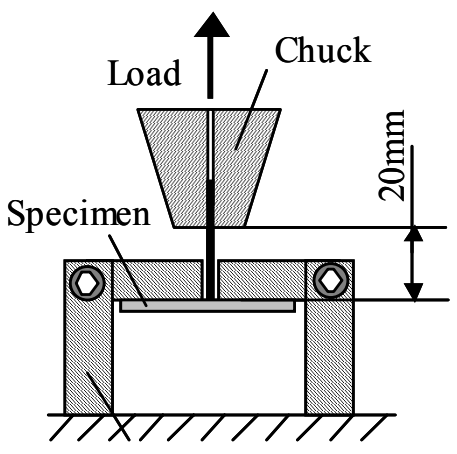

Instrument

(b) Pull-out testing method

Fig. 3 Schematic illustration of pull-out test.

To evaluate the yarn/matrix and matrix/coated-layer interface strengths, the yarn pull-out test was performed. The test is one of the mechanical testing methods for evaluating the interface strength, and it was carried out at a crosshead speed of $1.0 \mathrm{~mm} / \mathrm{min}$ using a universal testing machine and a special test jig tool, as shown in Fig. 3(b). The apparent interfacial shear strength (IFSS), $\tau_{\text {app }}{ }^{(12)}$, can be calculated using

$\tau_{\text {app }}=\frac{F_{\max }}{\pi D L}$,

where $D$ is the diameter of the yarn and $L$ is the embedded fiber length. The maximum force $\left(F_{\max }\right)$ is the maximum value on the force-displacement curve recorded in the yarn pull-out test.

In all tensile and pull-out tests, 10 specimens were prepared and data from more than 6 specimens for each material were analyzed to characterize the mechanical properties.

To examine the initial surface of yarns/fibers and the fractured surface of the testing specimens after tensile and pull-out tests, a scanning electron microscope (KEYENCE Corporation, VE8800) was used in this study.

\section{Experimental results and discussion}

\subsection{Tensile properties of composite and its constituents}

Figure 4 shows the stress-strain curves of hemp yarns. In this figure, data from 10 specimens at fracture are indicated. The average tensile strength of hemp yarns is $395 \mathrm{MPa}$ and the elongation is $3.2 \%$. 


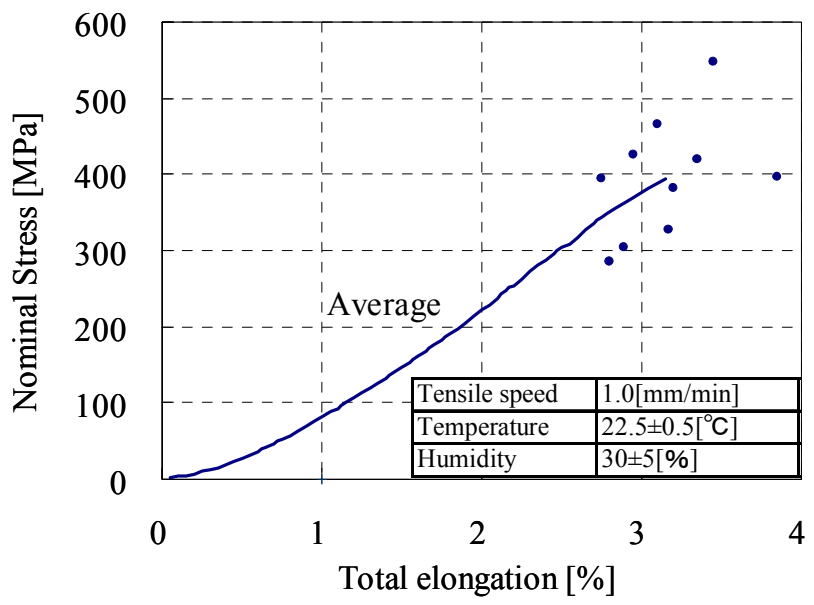

Fig. 4 Stress-strain curves of hemp bundle and data spread of tensile strength.

Figure 5 shows typical stress-strain curves of the PLA matrix, PVA coating layer, and composites with and without coating. In the figure, it can be seen that the average tensile strengths of composites with coating (THC) and without coating (UTHC) were 117 $\mathrm{MPa}$ and $98 \mathrm{MPa}$, respectively. Considering the scatter of data by error bar, we conclude that the coating of reinforcement yarns results in a strength enhancement of around $20 \%$. On the contrary, there is no significant difference between THC and UTHC.

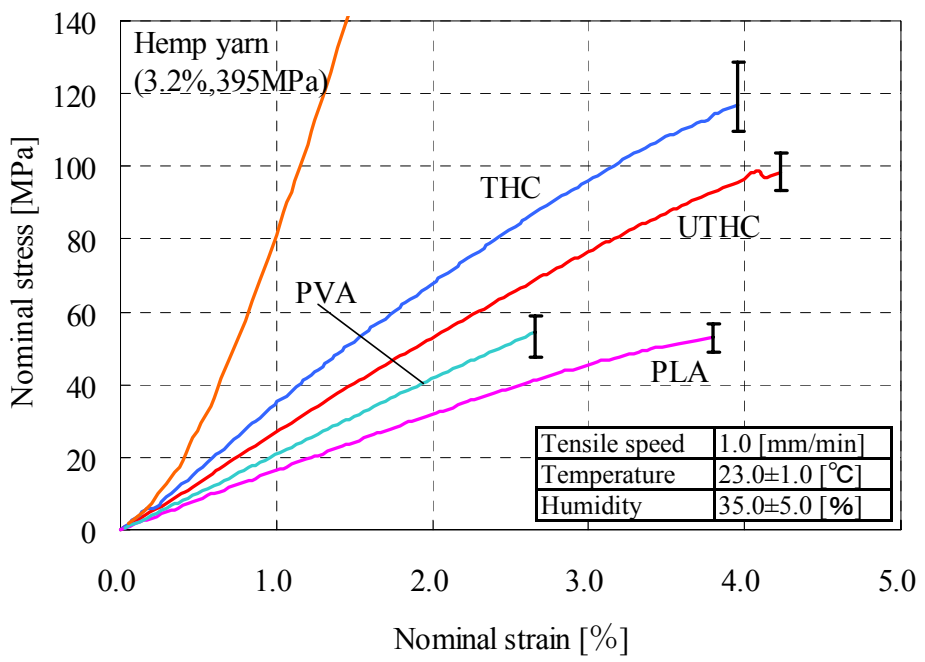

Fig. 5 Stress-strain curves of composites and their constituents.

\subsection{Interfacial characteristics of composite}

Figure 6 shows the load-displacement curves for THC and UTHC recorded in the yarn pull-out test. Using this figure, the interfacial adhesion status of the composites can be evaluated from the results of shear strength measurements. General trends for the composites were roughly observed: there is an initial increase in load with displacement, which is characteristic of elastic bonding at the yarn-matrix interface, up to the maximum debonding load, $F_{\max }$, beyond which the yarn pull-out process occurs in a stable fashion and shows a smooth transition. From these results, it is seen that the load of UTHC is higher than that of THC; the interfacial adhesion of UTHC is superior to that of THC so that the displacement of UTHC is greater as well.

The averaged IFSS values and their scattering ranges calculated under different interfacial conditions of the composites are presented in Fig. 7. The value of IFSS for THC 
is smaller by about $5 \mathrm{MPa}$ than that of UTHC. It is seen from the SEM observation that the pulling-out of fibers takes place on the surface of the coating. This result implies that although the PVA coating reduces the apparent interface strength, it results in an enhancement in the tensile strength of the composites.

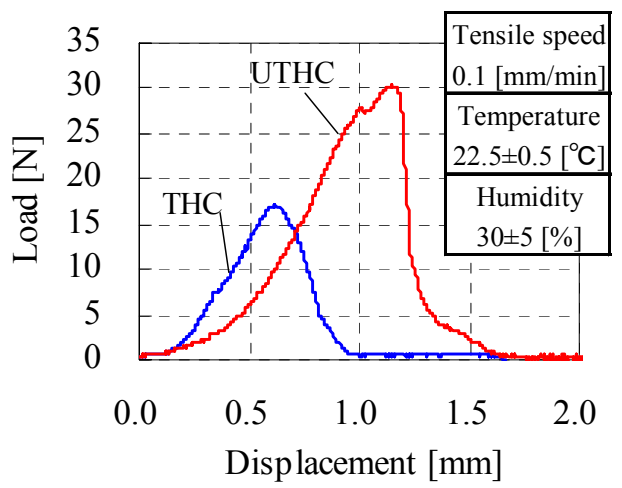

Fig. 6 Load-displacement curves obtained from the pull-out tests.

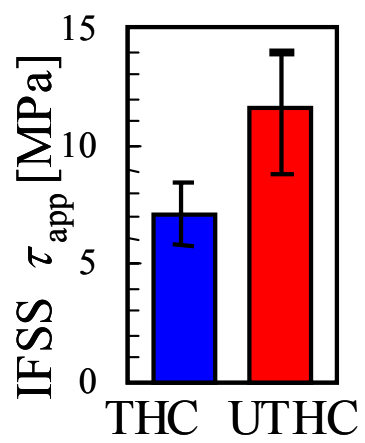

Fig. 7 IFSS measured in pull-out tests.

\subsection{SEM observation of fractured surface}

Figure 8 shows the micrographs of reinforcement yarn and coated surfaces prior to the pull-out test. After the coating process, it is seen that the yarn surface has become smooth overall. Figure 9 shows micrographs of the yarn and matrix after the pull-out test for noncoated and coated composites. For UTHC, Fig. 9(a) shows that a single yarn strand is stretched and has come loose. As seen in Fig. 9(b), fractured fine fibers remain attached to the fractured matrix surface. On the other hand, for THC, there is no difference from the initial coated yarn surface in Fig. 8(b), and no fiber or its tip remain around the bonded matrix hole.

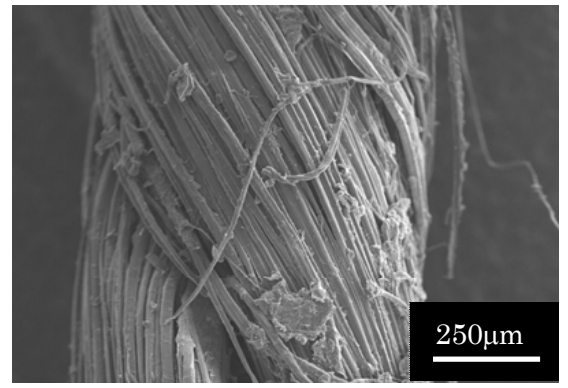

(a) Hemp yarn (UTHC)

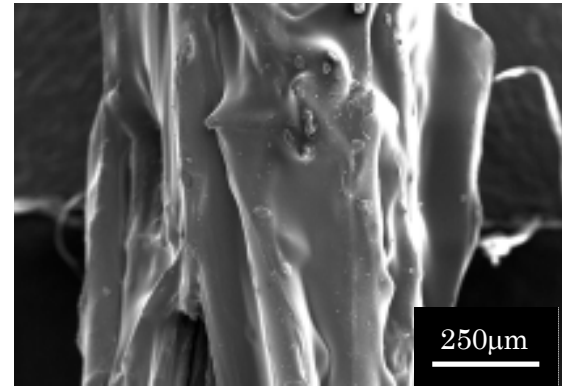

(b) Treated hemp yarn (THC)

Fig. 8 Micrographs of noncoated and coated hemp yarns prior to pull-out tests. 


\subsection{Effect of water-soluble resin coating on tensile characteristics}

In general, the stronger the interface strengths of the composites, the higher the stress transmission between the fiber/yarn and matrix, and thus the mechanical properties improve. However, compared with noncoated fiber bundles (UTHC), coated fiber bindles (THC) are considerably inferior in their apparent interfacial shear strength (IFCC). Nevertheless, THC is superior to UTHC in terms of tensile strength. This is because this water-soluble resin has an excellent impregnation characteristic among the yarns/fibers and plays the role of a binder, causing the yarns to behave as one fiber. We confirmed from microscopic observation that the fiber bundles were overall impregnated with water-soluble resin. If the shear stress applied at the interface of the matrix is considered to be transmitted to all fibers, then inter-yarn/fiber slipping does not occur. On the other hand, for UTHC, the water-soluble resin does not sufficiently impregnate into the yarns, and thus the nominal IFSS should be transmitted at only the outside surface of surrounding yarns. Therefore, at first, the fracturing of fibers takes place at only the outer interfacial part and then proceeds toward the inside; this is confirmed from the SEM images in Fig. 9(a). This fracture behavior can be understood from Fig. 6, which shows a drastic drop in the load after the peak load.

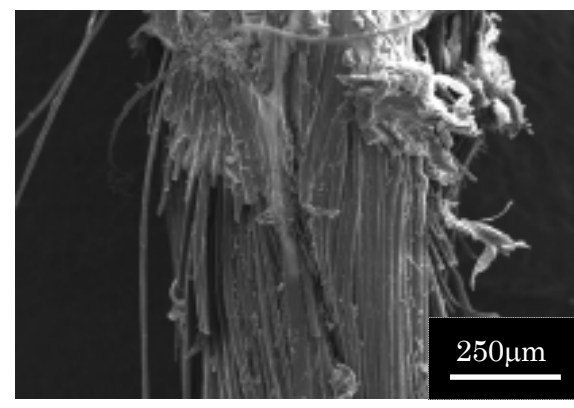

(a) Hemp yarn (UTHC) after fracture

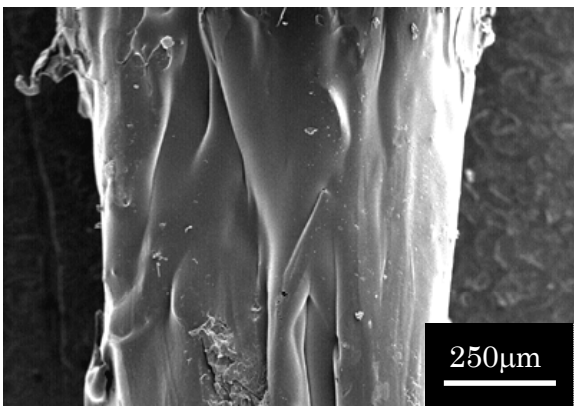

(c) Treated hemp yarn (THC) after fracture

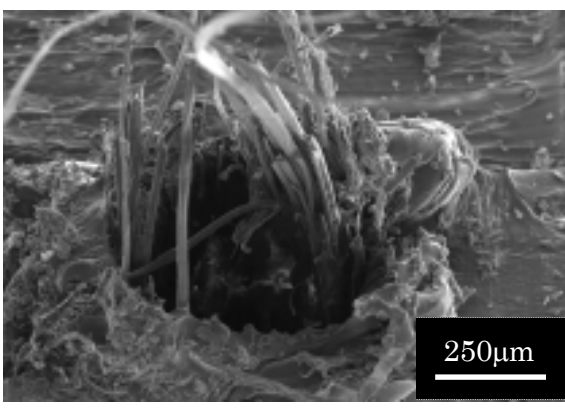

(b) Fractured surface at the matrix with hemp yarn (UTHC)

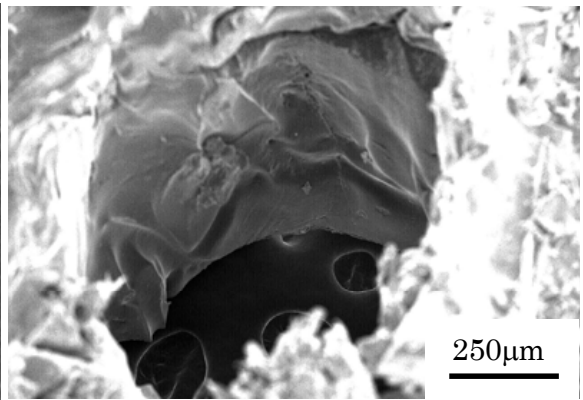

(d) Fractured surface at the matrix with treated hemp yarn (THC)

Fig. 9 Micrographs of yarn and matrix surfaces after fracture in the pull-out tests.

\section{Conclusions}

To fabricate high strength biodegradable green composites, we attempted to enhance the interface strength between the reinforcements and the matrix by coating the natural yarn reinforcements with a water-soluble biodegradable resin (PVA). Unidirectional biodegradable composites were made with hemp bundles used as the reinforcement and 
polylactic acid (PLA) used as the biodegradable resin matrix. The results obtained in this study are summarized as follows.

(1) It was demonstrated that the tensile strength of the green composite was enhanced by around $20 \%$ by coating a water-soluble resin onto the reinforcement fibers. Although the fiber volume fraction $V_{\mathrm{f}}$ was less than $30 \%$, the green composite had a tensile strength of $117 \mathrm{MPa}$. It is expected that when the fiber volume fraction is increased, the tensile properties of green composites would be further enhanced.

(2) For natural fiber bundles, the apparent interfacial shear strength between the coated bundle and PLA is inferior to that in the case of no coating. Nevertheless, water-soluble biodegradable resin possesses an excellent impregnation characteristic into the yarns so that it plays the role of a binder, resulting in an integrated yarn with noslippage between individual fibers. As a result, the apparent strength of the green composite improves.

\section{References}

(1) Wollerdorfer, M. and Bader, H., Influence of natural fibers on the mechanical properties of biodegradable polymers, Industrial Crops and Products, Vol.8 (1998), pp.105-112.

(2) Oksman, K., Skrifvars, M. and Selin, J-F., Natural fibres as reinforcement in polylactic acid (PLA) composites, Composites Science and Technology, Vol.63 (2003), pp.1317-1324.

(3) Keener, T.J., Stuart, R.K. and Brown, T.K., Maleated coupling agents for natural fibre composites, Composites Part A: Applied Science and Manufacturing, Vol.35 (2004), pp.357-362.

(4) Plackett, D., Andersen, T.L., Pedersen, W.B. and Nielsen, L., Biodegradable composites based on polylactide and jute fibres, Composites Science and Technology, Vol.63 (2003), pp.1287-1296.

(5) Okubo, K., Fujii, T. and Yamamoto, Y., Development of bamboo-based polymer composites and their mechanical properties, Composites Part A: Applied Science and Manufacturing, Vol.35 (2004), pp.377-383.

(6) Luo, S. and Netravali, A.N., Interfacial and mechanical properties of environment-friendly green composites made from pineapple fibers and poly (hydroxybutyrate-co-valerate) resin, Journal of Materials Science, Vol.34 (1999), pp.3709-3719.

(7) Nishino, T., Hirao, K., Kotera, M., Nakamae, K. and Inagaki, H., Kenaf reinforced biodegradable composite, Composites Science and Technology, Vol.63 (2003), pp.1281-1286.

(8) Herrera-Franco, P.J. and Valadez-Gonza'lez, A., Mechanical properties of continuous natural fibre-reinforced polymer composites, Composites Part A: Applied Science and Manufacturing, Vol.35 (2004), pp.339-345.

(9) Mohanty, A.K., Wibowom A., Misra, M. and Drzal, L.T., Effect of process engineering on the performance of natural fiber reinforced cellulose acetate biocomposites, Composites Part A: Applied Science and Manufacturing, Vol.35 (2004), pp.363-370.

(10) Ochi, S., Development of high strength biodegradable composites using Manila hemp fiber and starch-based biodegradable resin, Composites Part A, Vol.37 (2006), pp.1879-1883.

(11) Dauda, M., Yoshiba, M., Miura, K. and Takahashi, S., Processing and mechanical property evaluation of maize fiber reinforced green composites, Advanced Composite Materials, Vol.16, No.4 (2007), pp.335-347.

(12) Wong, S., Shanks, R.A. and Hodzic, A., Effect of additives on the interfacial strength of poly(L-lactic acid) and poly(3-hydroxy butyric acid)-flax fibre composites, Composites Science and Technology, Vol.67(2007), pp.2478-2482. 